\title{
Análisis de los costos de las aerolíneas
}

\section{Analysis of airlines costs}

\author{
Jessica Fernanda Moreno Ayala. ${ }^{1}$
}

DOI: . https://doi.org/10.33262/visionariodigital.v3i3.856

\begin{abstract}
The airlines constitute one of the fundamental pillars of the air transport system, being the producers of the industry and for the consumers (passengers and air cargo) it is of vital importance to know what costs influence in their daily activity, on the part of the airlines to know its situation and based on this, to propose strategies to attract more travelers, reducing the costs if possible to also decrease the Yield of the airline and with it the prices of the tickets for the passengers. In this document we will analyze the different business models currently handled by airlines, as well as the historical evolution of the airline's operating costs, highlighting the costs of: flight operations, maintenance, sales and promotion, amortizations, rights of use and assistance on land and passenger service. Within the costs of flight operations highlights the fuel cost that has been taking greater importance over time, becoming currently the most important cost for an airline, which must be analyzed in detail to try to control it and forecast it as much as possible. Exactly possible.
\end{abstract}

Key words: Airlines, costs, fuel, personal, maintenance, sales, historical evolution

\section{Resumen}

Las aerolíneas constituyen uno de los pilares fundamentales del sistema de transporte aéreo, siendo los productores de la industria y para los consumidores (pasajeros y carga aérea) es de vital importancia conocer que costes influyen en sus actividad diaria, por parte de las aerolíneas para conocer su situación y en base a ello plantear estrategias para atraer a más viajeros,

\footnotetext{
${ }^{1}$ Escuela de Gestión del Transporte de la Facultad de Administración de Empresas de la Escuela Superior Politécnica de Chimborazo, jessfma@ hotmail.com
} 
disminuyendo los costos de ser posible para disminuir también el Yield ${ }^{2}$ de la aerolínea y con ello los precios de los boletos para los pasajeros. En el presente documento se analizarán los diferentes modelos de negocios que manejan las aerolíneas en la actualidad, así como la evolución histórica de los costes operativos de la aerolínea destacando los costes de: operaciones de vuelo, mantenimiento, ventas y promoción, amortizaciones, derechos de uso y asistencia en tierra y servicio a pasajeros. Dentro de los costes de operaciones de vuelo destaca el coste combustible que ha ido tomando mayor importancia con el pasar del tiempo, constituyéndose en la actualidad como el coste más importante para una aerolínea, que debe ser analizado con detalle para intentar controlarlo y pronosticarlo lo más exacto posible.

Palabras clave: Aerolíneas, costos, combustible, personal, mantenimiento, ventas, evolución histórica.

\section{Introducción}

El Mercado de Transporte Aéreo es una industria cada vez más competitiva y demanda la constante actualización y manejo de distintas estrategias de los actores que lo componen. Dentro de este amplio mercado las aerolíneas desempeñan un papel fundamental siendo los transportistas, y, por lo tanto, el nexo entre las autoridades aeronáuticas nacionales e internacionales, los fabricantes, proveedores de servicios de navegación aérea y los pasajeros. El transporte aéreo siempre se ha caracterizado por poseer un alto nivel de regulación por parte del estado (1) y esto implica también una regulación de tarifas para los servicios de transporte aéreo brindado por las aerolíneas. La regulación de las tarifas aéreas lo establece $\operatorname{IATA}^{3}$ (2) y en el Ecuador también la DGAC ${ }^{4}$, establecido en el Art. 6 de la Ley de Aviación Civil (3) en donde se indica la obligación de la autoridad aeronáutica nacional de registrar las tarifas aéreas de pasajeros, aerolíneas nacionales y extranjeras, así como las de carga.

Siendo las aerolíneas una parte esencial en el mercado de transporte aéreo es de gran interés conocer la estructura de costes que éstas poseen, puesto que, los precios de los boletos aéreos que adquieren los usuarios de este medio de transporte dependen en gran medida de ello (4). En el presente artículo se efectuará un análisis de los principales costos de una aerolínea.

\footnotetext{
${ }^{2}$ Ingreso promedio de la aerolínea por pasajero

${ }^{3}$ International Air Transport Asociation. Asociación Internacional de Transporte Aéreo

${ }^{4}$ Dirección General de Aviación Civil
} 
Es necesario definir la palabra costo: puede significar la suma de esfuerzos y recursos que se han invertido para producir algo; otra definición puede ser que es un sistema de información que clasifica, acumula, controla y asigna los costos para determinar los costos de actividades, procesos y productos, y con ello facilitar la toma de decisiones, la planeación y el control administrativo. (5)

Los costos además tienen ciertas características que deben ser analizadas por cualquier empresa, en particular para el caso de estudio, por las aerolíneas: el análisis del costo es aproximado, esto implica que dos aerolíneas no son iguales y por lo tanto cada caso es especial; el análisis del costo es específico, depende de las condiciones del mercado particular y el tiempo; el análisis de costo es dinámico, la actualización de costos es fundamental por el avance tecnológico y la necesidad de nuevas adquisiciones; el análisis de costo puede elaborarse inductiva o deductivamente, dependiendo el enfoque puede analizarse de manera global o particular; el costo está precedido de costos anteriores y éste a su vez es integrante de costos posteriores, la línea del tiempo es esencial para un correcto análisis de costos. (6)

\section{Materiales y Métodos}

Los materiales utilizados en la presente investigación incluyen el uso de bases de datos de las principales organizaciones de transporte aéreo del mundo: $\mathrm{OACI}^{5}$, IATA, $\mathrm{ACI}^{6}$; se ha tenido en cuenta también la normativa vigente y disponible en la biblioteca de la autoridad aeronáutica nacional (DGAC). (7)

Para realizar el análisis de los costos que componen la operación diaria de las aerolíneas se emplearán distintos métodos de análisis:

\subsection{Método Deductivo}

Aristóteles y sus discípulos implantaron el razonamiento deductivo como un proceso del pensamiento en el que de afirmaciones generales se llega a afirmaciones específicas aplicando las reglas de la lógica. Es un sistema para organizar hechos conocidos y extraer conclusiones. (8). Para este caso en particular se partirá de la clasificación general de los costos de las aerolíneas: Operacionales y no operacionales, para después pasar a un análisis particular de cada uno de ellos, desglosando cada apartado que compone estos dos grandes grupos y

\footnotetext{
5 Organización de Aviación Civil Internacional

${ }^{6}$ Airport Council International. Consejo Internacional de Aeropuertos
} 
finalmente obtener una ponderación porcentual que indique el peso de cada costo en el balance general de costos de las aerolíneas.

\subsection{Método Inductivo}

Las conclusiones del razonamiento deductivo serán verdaderas sólo si las premisas en que se basan también lo son (8). Para aplicar la definición de método inductivo a la investigación es necesario conocer otra aclaración que hace Dávila (8): El método inductivo se conoce como experimental y sus pasos son: 1) Observación, 2) Formulación de hipótesis, 3) Verificación, 4) Tesis, 5) Ley y 6) Teoría.

Partiendo de las definiciones de Dávila para el análisis de los costos de las aerolíneas es necesario analizar varios factores que componen los costos de las aerolíneas, entre los principales tenemos: el precio de combustible en la aviación, modelos de negocio de las aerolíneas, estrategias comerciales, entre otros.

\subsection{Comparación cualitativa y cuantitativa}

La evaluación cualitativa se refiere a comparar una misma característica o cualidad entre distintas empresas (aerolíneas) en base a un criterio de valoración subjetivo y establecer un orden de menor a mayor o viceversa para ordenar las empresas analizadas.

La evaluación cuantitativa por el contrario consiste en evaluar distintas empresas en base a un criterio numérico y establecer un orden en función del valor numérico obtenido por cada empresa en cada criterio.

El conjunto de los dos análisis brinda un panorama global de la valoración que cada empresa ha obtenido y las ventajas competitivas que puede tener cada uno de ellas. Adicionalmente en este trabajo se realizará un análisis para ponderar los principales costos de las aerolíneas, basándose en dos criterios de evaluación: el porcentaje que representa cada coste principal en el total de las aerolíneas en general, y como varía la denominación del coste principal en función del modelo de negocio de cada aerolínea.

\subsection{Evolución temporal}

El transporte aéreo comercial tuvo su origen con el vuelo de Tampa - Florida Airbot, considerada la primera aerolínea comercial conectando en 1914 las ciudades de Tampa y Florida en Estados Unidos; sin embargo, las aerolíneas no empezarían a operar grandes vuelos comerciales sino a partir de la segunda guerra mundial donde empezaron a aparecer los primeros modelos de negocio de las aerolíneas, dando origen al modelo tradicional. Con el 
pasar del tiempo, el incremento de la competencia con la aparición de nuevas aerolíneas y la liberalización del mercado de Transporte Aéreo generó la aparición de nuevos modelos de negocio con los cuales las aerolíneas empezaron a desempeñar sus actividades: chárter, regionales y a partir de la década del 90 con gran fuerza hizo su entrada el modelo de negocio low cost.

Como se menciona en varios textos referentes a transporte aéreo a partir de la década de los 50 el costo más importante para las aerolíneas era el combustible, seguido de los costos devenidos por los pagos a tripulaciones. De este modo es necesario efectuar un análisis integral y detallado, haciendo uso de las técnicas mencionadas anteriormente; así como también un análisis temporal para evaluar la evolución de la ponderación de los principales costos en función de la década y el modelo de negocio de cada aerolínea.

\subsection{Modelos de negocio de las aerolíneas}

Para poder realizar un análisis completo de los principales costos y el porcentaje que cada uno de ellos representa en la totalidad de los egresos de una aerolínea en el mercado de transporte aéreo actual, es fundamental que se realice una diferenciación respecto del modelo de negocio con el que cada empresa aérea funciona. En la actualidad podemos distinguir claramente cuatro modelos distintos de negocio dentro de la industria aeronáutica:

\subsubsection{Modelo de negocio tradicional}

El modelo de negocio tradicional es el más antiguo de todos, empezó su desarrollo a partir de compañías de gobierno, conocidas también como aerolíneas de bandera o "full service ${ }^{7}$ que operaban bajo la tutela y gestión del gobierno nacional a partir de la segunda guerra mundial. En ese entonces el mercado de transporte aéreo era prácticamente un monopolio en el que existía una aerolínea por país y eran las únicas que cubrían las rutas existentes, nacionales e internacionales.

Este modelo de negocio fue evolucionando de tal manera que en el mundo ya muy pocas compañías operan con este modelo de negocio como empresa pública. Las aerolíneas consideradas como tradicionales hoy en día son empresas privadas, conformadas por la fusión del capital de varios inversionistas e incluso operan en colaboración con otras, en las mundialmente conocidas alianzas (One World, Star Alliance y Sky Team).

${ }^{7}$ Servicio completo a bordo de la aeronave 
Las aerolíneas agrupadas en este modelo de negocio se caracterizan por dar un servicio completo a sus pasajeros, es decir, incluyen en el valor del pasaje los servicios de: transporte, alimentación, facturación, manejo de equipaje, asistencia a bordo; además presentan una diferenciación de tarifas en cabina, permitiendo a los pasajeros acceder a servicios más personalizados en función del precio pagado por el boleto. Otra característica de las aerolíneas tradicionales es que operan utilizando el modelo de Hub and Spoke o centros de distribución, lo que les permite diversificar la cantidad de rutas ofrecidas concentrando vuelos de corto y medio radio en aeropuertos de gran tamaño para abastecer de pasajeros a los vuelos de largo radio.

Para comprender mejor el modelo de negocio tradicional, se citan algunos ejemplos de aerolíneas que mantienen esta forma de operación: TAME, Avianca, American Airlines, LATAM, Lufthansa, KLM, Emirates, Qantas, entre otras.

\subsubsection{Modelo de negocio regional}

El modelo de negocio regional en las aerolíneas nació en la década de los 70, cuando los gobiernos y administraciones estatales tuvieron la necesidad de conectar varios territorios separados por grandes accidentes geográficos o territorios insulares, a los cuales se podía acceder únicamente a través del transporte aéreo.

Las aerolíneas regionales operan con aeronaves de menor tamaño, recorriendo distancias menores (hasta $2500 \mathrm{~km}$ ) y sin brindar muchos servicios a los pasajeros en cabina. Actualmente este tipo de aerolíneas pueden operar de dos maneras: bajo su propia marca; es decir, de manera independiente operando rutas cortas y con aeronaves de máxima capacidad de 100 pasajeros, o como una aerolínea alimentadora, en este caso la aerolínea regional es una filial de una aerolínea tradicional y cumple la función de recolectar a los pasajeros de lugares lejanos inaccesibles y llevarlos a la base de operaciones de una aerolínea más grande para alimentar vuelos de medio y largo radio. Dentro de los ejemplos destacados podemos mencionar a Lufthansa regional, KLM Cityhopper, Air Nostrum, Azul, entre otras.

\subsubsection{Modelo de negocio chárter}

El modelo chárter consiste en vuelos de transporte aéreo no regular, por lo tanto, no tienen rutas ni frecuencias pre establecidas como otras aerolíneas. Una aerolínea chárter trabaja a demanda, bajo los requerimientos del cliente. Los vuelos chárteres son contratados con la anterioridad 
que el cliente elija, siendo el tiempo mínimo de aviso, 72 horas previas a la realización del vuelo.

El modelo de negocio tipo chárter se proliferó en el mercado del transporte aéreo a partir de la década de los 80 y tuvo gran acogida hasta finales del año 2000, junto con la gran oleada turística que tuvo lugar en esa época. El vuelo chárter se ofrece comúnmente como parte de un paquete turístico que suele ser comercializado por agencias de viaje, otra de las modalidades del chárter es cuando se contrata para una delegación deportiva, artística o algún ejecutivo. En Ecuador los vuelos de tipo chárter están regulados por la resolución No. 003/2015 emitida en marzo de 2015.

Los costos de las aerolíneas chárter pueden ser esencialmente operativos o elevarse a niveles extraordinarios en función de los requerimientos del cliente, por lo general, en estos casos el cliente envía la lista de sus necesidades y en función de ello la aerolínea emite una proforma y finalmente se llega o no a un acuerdo entre las partes. En la actualidad los vuelos chárter completos son menos comunes, en su lugar se utiliza el chárter parcial, en esta modalidad las aerolíneas (tradicionales, regionales o low cost) venden una cantidad de asientos de uno de sus vuelos regulares a una agencia de viajes para que sean comercializados en el contexto de un paquete turístico.

A nivel mundial quedan ya muy pocas aerolíneas puramente chárteres, una de ellas es Monarch, en el Reino Unido que está especializada en comercializar vuelos de este tipo y ofrece una variedad bastante amplia de rutas a sus clientes, conectando el Reino Unido casi con todas las ciudades del mundo, según el cliente lo decida.

\subsubsection{Modelo de negocio low cost}

El modelo low cost es uno de los más nuevos en el mercado de transporte aéreo y basa su estrategia en disminuir al máximo los costes operativos utilizando distintas estrategias como: utilizar una flota joven y así disminuir costos de mantenimiento, usar medios digitales para disminuir costes de promoción y ventas, reducir servicios a bordo para los pasajeros. El disminuir estos costos les permiten a las aerolíneas bajo este modelo de negocio abaratar los precios de los boletos aéreos pues básicamente este tipo de aerolíneas cobran por el servicio de transporte entre dos aeropuertos sin incluir servicios extra.

La comida a bordo, el equipaje, facturación en el aeropuerto, la posibilidad de elegir la ubicación del asiento en el avión son rubros por los que las aerolíneas low cost cobran extra a 
sus pasajeros; sin embargo, costos como el combustible están un poco fuera del control de los directivos de estas aerolíneas y es aquí donde las estrategias del departamento de compras de estas aerolíneas cobran mucha importancia para generar estrategias viables para la reducción de costes en general.

Una de las aerolíneas emblemas en este modelo de negocio es la irlandesa Ryanair, que ha entendido el modelo de negocio low cost muy bien, llegando a considerarse entre los expertos del transporte aéreo un modelo ultra low cost que abarata mucho los costes operacionales y permite a la aerolínea ofrecer boletos de avión muy baratos, permitiéndole por cinco años consecutivos posicionarse en el cuarto lugar de las aerolíneas con mayores ingresos anuales solo por debajo de las emblemáticas: American Airlines, Delta Airlines y Emirates Airlines.

\subsection{Clasificación de los costos de las aerolíneas}

Para analizar los costos de las aerolíneas y su evolución es necesario conocer la clasificación de los diferentes costos que están presentes en la operación diaria de una aerolínea, a continuación, se explican los costes de una aerolínea, según la clasificación realizada por OACI. (8):

\subsubsection{Costos Operativos}

Los costos operativos de una aerolínea son todos aquellos que están relacionados directamente con el cuore ${ }^{8}$ del negocio de las aerolíneas, es decir, su operación. Engloban todos aquellos costos necesarios para poner en funcionamiento la aeronave y dar el servicio de transporte a los pasajeros, con todos los requerimientos que estos implican.

A su vez los costos operativos se subdividen en directos e indirectos y cada uno de ellos a su vez en fijos y variables.

Los costos operativos directos son aquellos sin los cuales la aerolínea no podría operar, ni ofrecer servicios de transporte. Dentro de estos podemos mencionar algunos importantes como el combustible, el mantenimiento, pago a tripulaciones, pagos de tasas aeroportuarias, entre otros.

Los costos operativos directos se dividen en fijos y variables. Dentro de los costos fijos se pueden nombrar: amortizaciones, pagos de seguros aéreos, alquileres fijos, entre otros;

${ }^{8}$ Corazón. Lo esencial de un negocio. 
mientras que en los costos variables se pueden mencionar: el combustible, pagos de tripulación, tasas aeroportuarias, pagos por prestación de servicios de navegación aérea. Los costos operativos indirectos por otra parte constituyen pagos que son necesarios efectuar que complementan las necesidades básicas de las aerolíneas, su valor viene dado por el pago que efectúa la aerolínea más una variante debido a las condiciones de contorno propias de cada mercado de transporte aéreo, así como dependen del modelo de negocio que cada aerolínea maneje para su desempeño. Dentro de los principales costos operativos indirectos se pueden mencionar los siguientes: ventas, promociones, servicio al pasajero, escalas, compensaciones a los pasajeros en casos de retrasos o cancelaciones de vuelos.

\subsubsection{Costos no operativos}

Los costos no operativos de la aerolínea son aquellos que no se consideran esenciales a la actividad principal de la aerolínea, sin embargo, son complementarios. Los costos no operativos constituyen una parte fundamental en una aerolínea sobre todo por la cantidad de dinero que generan en ingresos, luego de haber sido considerados en los gastos. Los principales costos no operativos que se pueden mencionar son: intereses, resultados de activos, resultados de filiales, diferencias de cambio.

Merecen mención especial dentro de los costos no operativos los resultados de filiales, mismos que se obtienen en el caso por ejemplo de los grupos comerciales que se forman actualmente entre aerolíneas, como el grupo IAG que agrupa a Iberia, British Airways y Vueling.

Dentro de los costos operativos de una aerolínea se tiene el costo de combustible, el mismo que es esencial en el balance general de una aerolínea y sin duda es parte importante a la hora de determinar el precio de un boleto de avión para los pasajeros. El combustible se considera un costo fijo y a la vez variable; una parte es fija debido a que se adquiere con suficiente anticipación en el mercado de futuros a un precio fijo en base al costo del barril de petróleo en ese momento y la proyección que se realiza del mismo, esto puede generar dos escenarios en el futuro de la aerolínea: que las proyecciones hayan subestimado el valor del petróleo y con ello del combustible y por lo tanto la aerolínea ha ahorrado en la compra de ese combustible, o por el contrario que se haya calculado un precio más alto y con ello la aerolínea haya pagado un sobreprecio por ese combustible. En cualquiera de las dos situaciones anteriores el monto pagado por la aerolínea de esa cantidad de combustible 
se considera fijo para el período actual, puesto que el dinero en la mayoría de los casos ya suele ser desembolsado con anterioridad.

Por otra parte, es imposible adquirir con la anticipación suficiente y en las cantidades requeridas todo el combustible que una aerolínea necesita para un período de tiempo (usualmente un semestre o un año), por lo que la otra parte del combustible que se compra en el período presente de la aerolínea se considera un coste fijo.

\section{Resultados y Discusión}

Por lo mencionado anteriormente es indispensable conocer la evolución histórica del precio del petróleo, a continuación, se muestra esta evolución en la última década (9):

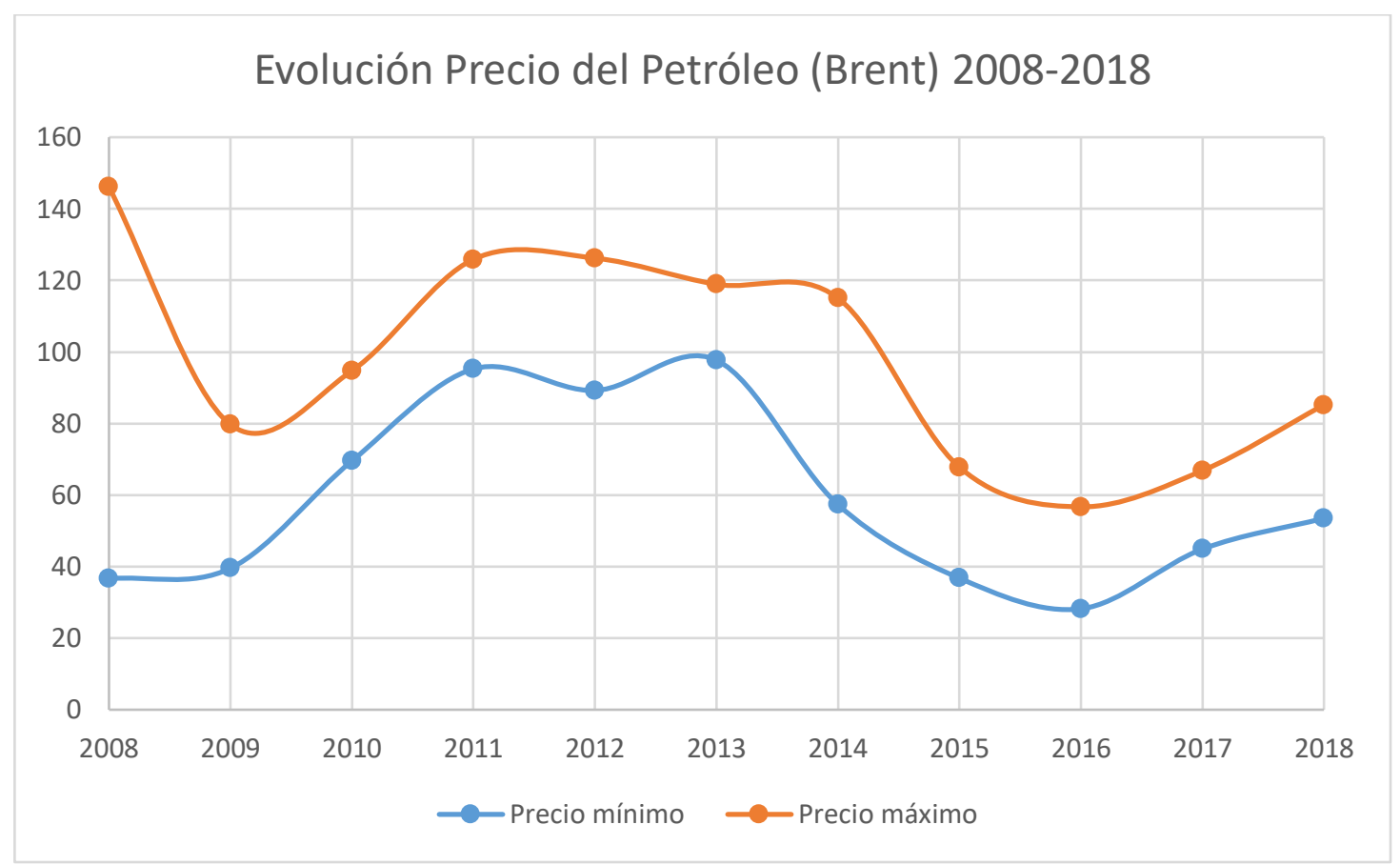

Figura 1. Evolución del precio del petróleo

En las siguientes tablas y organizadores gráficos se presentan los resultados de los porcentajes que históricamente representaron los principales costes de las aerolíneas y los porcentajes actuales que representan cada uno de los costes en la actualidad, teniendo en cuenta los dos principales modelos de negocios que se tienen de aerolíneas (tradicionales y low cost) obtenidos de la investigación de acuerdo a las técnicas mencionadas con anterioridad de los costos de las aerolíneas. 
Tabla 1. Evolución de los costos operativos

\begin{tabular}{lcccc}
\hline \multicolumn{1}{c}{ Rubro/Año } & $\mathbf{1 9 9 3}$ & $\mathbf{2 0 0 2}$ & $\mathbf{2 0 1 0}$ & $\mathbf{2 0 1 8}$ \\
\hline Combustible & 12 & 12,9 & 25 & 27 \\
Seguro de aviones & 0,4 & 0,5 & 0,5 & 1,2 \\
Tripulación técnica & 7,4 & 9 & 9,2 & 9,5 \\
Alquileres & 5,6 & 6,9 & 2,1 & 2,3 \\
Entrenamiento de tripulaciones & 0,5 & 0,2 & 0,2 & 0,2 \\
Otros & 0,6 & 1,1 & 0,25 & 0,1 \\
Operaciones de Vuelo & $\mathbf{2 6 , 5}$ & $\mathbf{3 0 , 6}$ & $\mathbf{3 7 , 2 5}$ & $\mathbf{4 0 , 3}$ \\
Mantenimiento & $\mathbf{1 0 , 1}$ & $\mathbf{1 1 , 3}$ & $\mathbf{8 , 5}$ & $\mathbf{8 , 2}$ \\
Amortización aviones & 5 & 5,4 & 4,1 & 3,1 \\
Amortización equipos de tierra & 1,6 & 1,4 & 0,2 & 0,15 \\
Resto & 0,4 & 0,3 & 0,05 & 0,05 \\
Amortizaciones & $\mathbf{7}$ & $\mathbf{7 , 1}$ & $\mathbf{4 , 3 5}$ & $\mathbf{3 , 3}$ \\
Ayudas a la navegación & 2,4 & 2,4 & 2,7 & 2,8 \\
Derechos de aterrizaje & 4,1 & 4 & 5,3 & 5,1 \\
Escalas & 10,8 & 10,6 & 10,5 & 10,4 \\
Derechos de uso y asistencia en tierra & $\mathbf{1 7 , 3}$ & $\mathbf{1 7}$ & $\mathbf{1 8 , 5}$ & $\mathbf{1 8 , 3}$ \\
Servicios pasajeros & $\mathbf{1 0 , 5}$ & $\mathbf{1 0 , 3}$ & $\mathbf{1 2 , 1}$ & $\mathbf{1 2 , 2}$ \\
Venta y promoción & $\mathbf{1 6 , 4}$ & $\mathbf{1 0 , 7}$ & $\mathbf{9 , 5}$ & $\mathbf{9 , 3}$ \\
Generales y administrativos & $\mathbf{7 , 1}$ & $\mathbf{7 , 1}$ & $\mathbf{6 , 5}$ & $\mathbf{6 , 1}$ \\
Otros & $\mathbf{5 , 1}$ & $\mathbf{5 , 9}$ & $\mathbf{3 , 3}$ & $\mathbf{2 , 3}$ \\
\hline
\end{tabular}

Fuente: Autor

Figura 2. Grafico comparativo del aumento de los costos operacionales
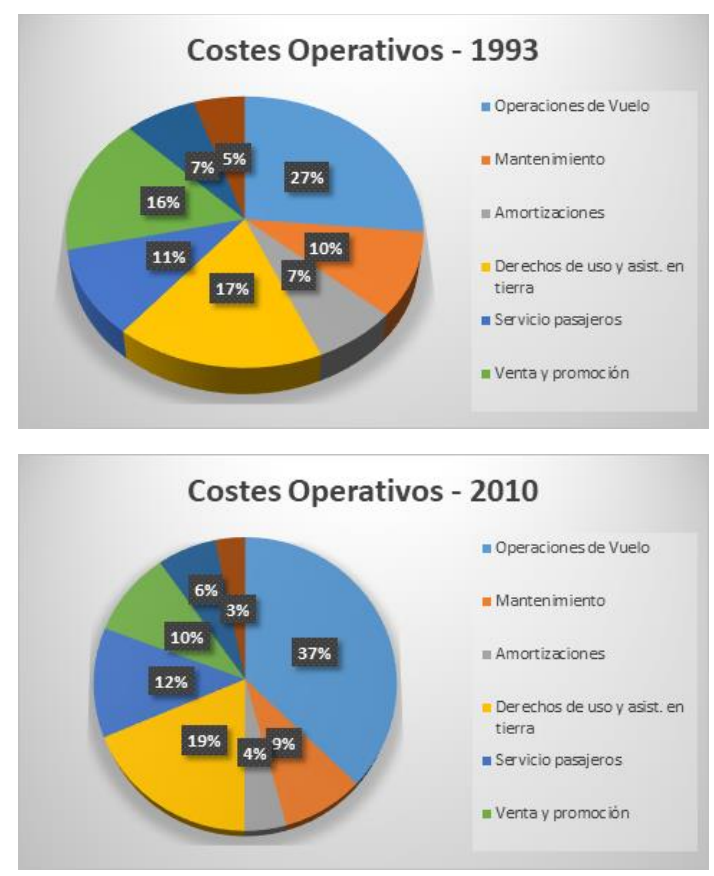

Fuente: Autor
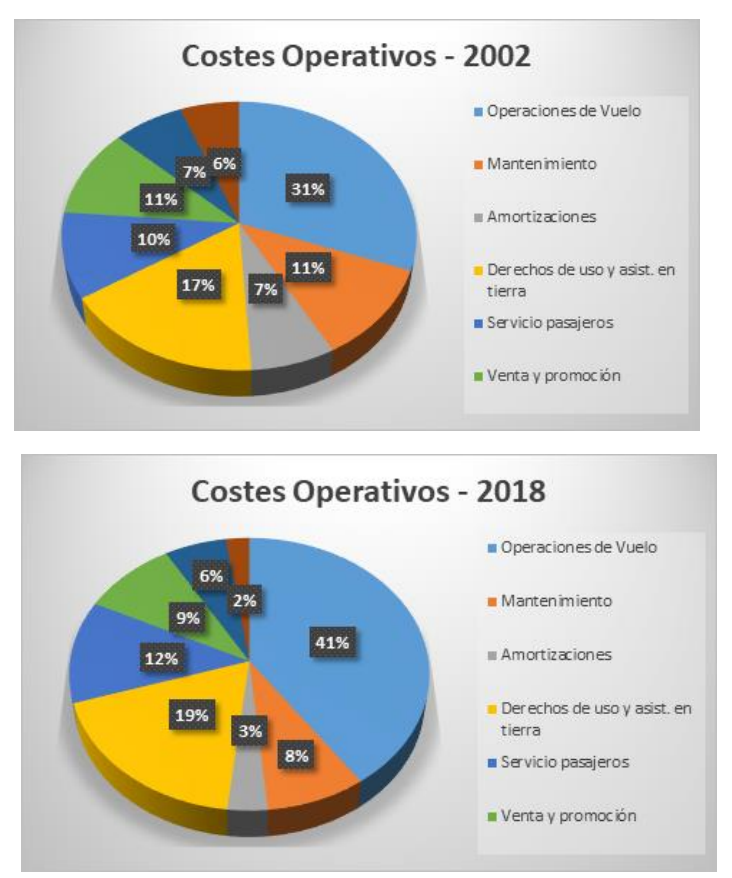
Se puede observar de la tabla y los gráficos anteriores como han ido evolucionando los costes de las aerolíneas que, a pesar de la disminución del precio del barril de petróleo, el coste de combustible se ha vuelto más representativo en el total de los costes de las aerolíneas haciendo que las operaciones de vuelo representen el $41 \%$ de los costes totales, siendo el combustible un $27 \%$ del total.

Por otra parte se puede decir que los costes de tripulación técnica y servicio a pasajeros han aumentado, esto puede estar justificado por el aumento de flota de aeronaves y también el número creciente de pasajeros teniendo en cuenta que el transporte aéreo crece en promedio un 5\% anual; mientras que el coste de amortización de aeronaves así como el de ventas y promociones han disminuido, en este caso se puede atribuir a la proliferación de la promoción de productos haciendo uso del internet, las páginas web y las aplicaciones móviles de las aerolíneas y las alianzas han permitido abaratar costos y extender el mercado al que pueden acceder las aerolíneas.

\section{Conclusiones}

Las aerolíneas constituyen una parte muy importante del sistema de transporte aéreo, los transportistas de este modo de transporte se agrupan mediante la IATA que actualmente se encuentra constituida por un poco más de 1600 miembros alrededor del mundo. Los costes de las aerolíneas deben ser analizados con detenimiento pues constituyen la base mediante la cual se fija el costo del boleto de avión en cada una de las rutas que las aerolíneas operan.

Merece especial atención el costo del combustible, que es un coste que posee una parte fija y una parte variable y su porcentaje sobre el total de los costes que ha se ha ido incrementando con los años, pasando de tener una participación del 12\% aproximadamente a un $28 \%$ el año anterior aun cuando el costo del barril de petróleo ha ido disminuyendo paulatinamente desde el repunte del precio en el año 2014 donde el barril de petróleo alcanzó valores cercanos a los $\$ 120$.

De la misma manera se analizó la evolución de otros costes importantes como el mantenimiento, la tripulación especializada, amortizaciones, venta y promociones; los mismos que han ido cambiando con el pasar del tiempo. Para el año 2018 los costes de ventas y promoción alcanzaron el 9,3\% del total motivado por el mayor uso de canales no convencionales de ventas como el internet. 


\section{Referencias}

(1) Oficina de Regulación Económica. Revisión y análisis de la estructura de costos de transporte aéreo. Bogotá D. C. 2009

(2) International Air Transport Asociation [Internet], Canadá: Montreal [actualizada en agosto de 2018; acceso 20 de agosto 2018]. [aprox. 3 pantallas]. Disponible en: http://www.iata.org/

(3) Dirección General de Aviación Civil [Internet]. Quito-Ecuador [actualizada en septiembre de 2018; acceso 01 de septiembre 2018]. [aprox. 6 pantallas]. Disponible en http://www.aviacioncivil.gob.ec/wp-content/uploads/downloads/2016/07/Ley-deAviacion-Civil.pdf

(4) Ginieis M, Sánchez MV, Campa F. Los costes en el sector del Transporte Aéreo. Una revisión de la literatura internacional. [Internet]. 2012 [citado 03 septiembre 2018]; Disponible en: http://www.observatorioiberoamericano.org/ricg/No_20/Mat\%C3\%ADas_Ginieis,_Victoria_Schez_Rebull,_Fern ando_Campa.pdf

(5) Robles Roman, Carlos Luis. Costos históricos

(6) Suárez, Carlos. Costo y tiempo en edificación. Editorial Limusa. México. 2005. Disponible en:

https://books.google.es/books?hl=es\&lr=\&id=f8G8UFFjd9sC\&oi=fnd\&pg=PA3\&dq=co stos+definiciones\&ots=goibB_el7P\&sig=KqXDyyankaLMfn_I2E69xoYuljw\#v=onepag $\mathrm{e} \& \mathrm{q}=\operatorname{costos} \% 20 \mathrm{definiciones} \& \mathrm{f}=$ false

(7) Organización de Aviación Civil Internacional [Internet], Canadá: Montreal [actualizada en septiembre de 2018; acceso 05 septiembre 2018]. [aprox. 4 pantallas]. Disponible en: http://www.icao.int

(8) Benito, A (2008). Los Aeropuertos en el Sistema de Transporte. $1^{\text {a }}$ ed. Madrid:

(9) Precio del Petróleo. [Internet]. 2019 [citado 10 febrero 2019]: Disponible en: https://www.preciopetroleo.net/precio-petroleo-2018.html

(10)Dávila G. El razonamiento inductivo y deductivo dentro del proceso investigativo en ciencias experimentales y sociales. [Internet]. 2006 [citado 10 septiembre 2018. 184-194. Disponible en: https://www.redalyc.org/pdf/761/76109911.pdf 


\section{PARA CITAR EL ARTÍCULO INDEXADO.}

Moreno Ayala, J. (2019). Análisis de los costos de las aerolíneas. Visionario Digital, 3(3), 313326. https://doi.org/10.33262/visionariodigital.v3i3.856

\section{Liencia}

El artículo que se publica es de exclusiva responsabilidad de los autores y no necesariamente reflejan el pensamiento de la Revista Visionario Digital.

El artículo queda en propiedad de la revista y, por tanto, su publicación parcial y/o total en otro medio tiene que ser autorizado por el director de la Revista Visionario Digital.
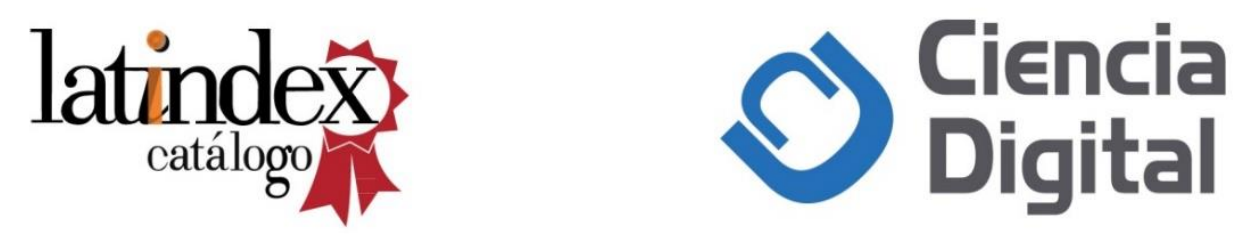